\title{
AUTOMATED OPTIMUM VISUALIZATION SYSTEM FOR CONSTRUCTION DRAWING READING
}

SUBMITTED: August 2021

REVISED: October 2021

PUBLISHED: October 2021

GUEST EDITORS: Francis Siu, Hung-Lin Chi, Hsi-hsien Wei \& Minkoo Kim

DOI: $10.36680 /$ j.itcon.2021.036

Jack Swanborough,

Hurstbourne Forge, SP10 3SL, UK;

jack@hurstbourneforge.co.uk

Min-Koo Kim, Dr,

Chungbuk National University, 28644, Korea;

joekim@chungbuk.ac.kr (corresponding author)

Eva Agapaki, Dr,

University of Florida, 32603, United States;

agapakie@ufl.edu

Ioannis Brilakis, Dr,

University of Cambridge, CB2 1PZ, UK;

ib340@cam.ac.uk

SUMMARY: The task of reading drawings on construction sites has significant efficiency and cost problems. Recent products utilising laser projectors attempt to address the issue of drawing comprehension by projecting full scale versions of the drawings onto $3 D$ surfaces, giving an in-place representation of the steps required to complete a task. However, they only allow projection in red or green at a single brightness level due to the inherent constraints of using a laser-based system, which could cause problems depending on the surface to be projected on and the ambient conditions. Thus, there is a need for a solution that is able to adjust the visualisation parameters of the displayed information based on the surface being projected onto. This study presents a system that automatically changes the visualisation parameters based on the colour and texture of the current surface to make drawings visible under any planar-like surfaces. The proposed system consists of software and hardware, and the software algorithm contains of two parts 1) the optimisation run that computes and updates the visualisation parameters and 2) the detection loop which runs continually and checks if the optimisation run needs to be triggered or not. In order to verify the proposed system, tests on 8 subjects with 4 background surfaces commonly found on site were performed. The test subjects were timed to find 10 bolt holes projected onto the surface using the optimisation system, which was then compared to a control case of black lines projected onto a white background. The system allowed users to complete the task on the real-world backgrounds in the same time as the control case, with the system resulting in up to a $600 \%$ decrease in recognition time on some backgrounds.

KEYWORDS: Drawing reading; construction sites; laser projection system; optimal visualization

REFERENCE: Jack Swanborough, Min-Koo Kim, Eva Agapaki, Ioannis Brilakis (2021). Automated optimum visualization system for construction drawing reading. Journal of Information Technology in Construction (ITcon), Special issue: 'CIB World Building Congress 2019: Constructing Smart Cities', Vol. 26, pg. 681-696, DOI: 10.36680/j.itcon.2021.036

COPYRIGHT: ( $) 2021$ The author(s). This is an open access article distributed under the terms of the Creative Commons Attribution 4.0 International (https://creativecommons.org/licenses/by/4.0/), which permits unrestricted use, distribution, and reproduction in any medium, provided the original work is properly cited. 


\section{INTRODUCTION}

It is well known that the construction sector and mechanical, electrical, and plumbing (MEP) trades in particular have comparatively low productivity compared to other industries. According to the UK Office of National Statistics (Office of National Statistics, 2021), productivity in the UK construction sector is 35-40\% lower than other industries such as agriculture and manufacturing, and this low productivity is attributed to the nature of the construction sector that is primarily affected by the productivity of individual workers. The construction industry relies heavily on both unskilled and skilled manual labour, and so the productivity of individual workers is a large proportion of the productivity of the sector as a whole. This individual performance is a direct result of their proportion of time spent on effective work, and for MEP trades in particular more time is spent on contributory tasks than effective work, (Oglesby et al., 1989) reducing their overall productivity. Contributory work for these trades includes tasks such as reading drawings, measuring and marking, (Yates, 2014) so reducing time spent on these tasks would be a direct benefit to the whole industry.

The task of reading drawings on the jobsite can be split into two parts: 1) accessing the drawing, and 2) using it to understand the steps required to complete the current task. Traditional methods using paper drawings have many obvious efficiency and cost problems, with keeping track of versions and distributing change orders being particularly difficult, especially on projects requiring large numbers of workers to have access to the same drawing. There are many existing market solutions that attempt to facilitate drawing access and collaboration between the jobsite and the design office. These solutions solve these issues to some extent. Software solutions such as Autodesk 360 (Autodesk 360, 2021) provide ways to instantly share up to date drawings between designers and workers, allowing effective communication and reducing rework resulting from incorrect information at the jobsite. Specialist hardware products such as the Trimble Rugged Tablet (Trimble, 2021) take this concept further, by not only facilitating up to date drawings access but allowing on site recording of measurements and other survey/inspection data. However, whilst these tools clearly make drawing access faster and more accurate and make collaboration easier, they require specialist skills and knowledge to operate effectively. In addition, they serve only to provide the correct drawing quickly without necessarily helping with understanding it.

Currently, products such as the Z-Laser range of laser projectors (Z-laser, 2021) attempt to address the issue of drawing comprehension by projecting full scale versions of the drawings onto any 3D surface, giving an in-place representation of the steps required to complete a task making the process more intuitive for workers. Unfortunately, these systems are highly optimised towards predictable and repeatable factory setups, not the highly changeable construction site. They are expensive and require complex alignment, setup and specially formatted drawings. Moreover, they only allow projection in red or green at a single brightness level due to the inherent constraints of using a laser-based system, which could cause problems depending on the surface to be projected on and the ambient conditions. In addition, there is currently no proposed solution to adjust the visualisation parameters of the displayed information based on the surface being projected onto. For example, orange lines projected onto a brick surface would not be visible to the user and so render the system ineffective for that situation. As the laser projection system has a wide variety of envisaged use cases, a system that could adapt automatically to any surface is desirable, and would add value to a final product.

The study aims to design a system that is able to automatically change the visualisation parameters such as line thickness and line colour based on the colour and texture of the current surface to make drawings visible under any planar-like surfaces. The organization of this paper is as follows. First, a review on related work and functional requirements required for a solution are presented in Section 2. Section 3 describes the system design of the proposed solution, including software and hardware. Validation tests and their results are presented in Section 4. Finally, Section 5 concludes with a short summary of this study.

\section{RESEARCH BACKGROUND}

\subsection{Related Work}

To bridge the gap between the functionality of the high-end solutions and the instant usability of the more common tools, there has been a large amount of research into using new methods, especially using Augmented Reality (AR) and new display technologies, to solve the problems associated with the construction drawing reading. Developing software systems that are able to be used on smartphones and tablets is of particular interest and often focus on use cases needing workers to manually align drawings to actual structures, providing an AR overlay of the drawing 
on images of the jobsite (Bae et al., 2013; Feiner et al., 1993). Recently, Wen et al. (2021) showed that AR methods assisted students to better understand complicated 2D drawings.

AR for the Architecture, Engineering and Construction (AEC) sector has been well documented by (Chi et al., 2013; Rankohi and Waugh, 2013; Wang et al., 2013). These methods have been extensively used for construction progress monitoring (Golparvar-Fard et al., 2004; Kim and Kang, 2017; Wen and Kang 2014), site planning (Singh and Delhi, 2018) or hazard identification, training and inspection (Li et al., 2018). Golparvar-Fard et al. (2004) reconstructed time-lapsed images to generate 4D as-built Building Information Models (BIM) and compared them with the as-designed 4D BIM models. Kim and Kang (2017) used static web cameras to generate images and then superimpose them on the physical site using a 4D simulation system for a railway construction project. Zollman et al. (2014) used images from UAV to generate point clouds and implemented this in a 4D simulation system for progress verification on site. Zaher et al. (2018) has added cost to generate a 5D model in Autodesk Navisworks and visualize it on mobile devices.

These applications mainly focus on individual use of the AR model and require a 3D BIM model for their AR system. Another limitation is that they have a relatively low precision if markers are occluded, which is not suitable for tasks like taking measurements and marking. Even if their accuracy could be improved, the applicability of these solutions is limited for the current problem as see-through and video-overlay AR systems provide no image on the surface itself, so there is no way to accurately transfer points into the real world. Some other limitations of these systems are: (1) they employ mobile devices or wearable head-mounted displays that can impede collaboration with other workers (Park et al., 2017) and (2) have relatively narrow field of view which ranges between 30 to 70 degrees diagonally (Low et al., 2001). The latter has a negative impact on human performance on navigation, spatial awareness and visual search tasks (Hubert, 1982; Alfano and Michel, 1990; Arthur, 2000).

\subsubsection{Projection based AR methods}

Projection based AR is far more useful in this regard, as an image is produced on the surface of interest. These methods have been widely investigated in the literature (Dalsgaard and Halskov, 2011; Bandyopadhyay et al., 2001; Siegl et al., 2015). These methods were originally used to apply textures, colours and patterns on buildings or walls (Raskar et al., 1998; Mine et al., 2012). Then, these evolved into studies deploying multiple projectors which were mounted on reconstructed walls and involved user interactions to paint the walls, however this implementation is specific to the environment setup that they used for their prototype (Low et al., 2001). A portable projection based AR system for interior design "Do It Yourself" users was implemented by Park et al. (2017), where users can design interiors in spaces that are not predefined. However, a prerequisite of their method is that the users need to have a 3D map of their space, which is not always the case in construction sites.

Projection based AR assisted systems have also been widely used by assembly workers in factories (Funk et al., 2017; Hou et al., 2013; Cuperschmid et al., 2016). These studies measured reduction in task completion time by $50 \%$ and fewer implementation errors. A laser projection system was developed to display images in an office environment to aid collaboration (Takahashi et al., 2011). Another study proposed a system that projects CAD drawings onto walls at the jobsite using a mobile device attached to a standard hard hat to remove the need for paper plans and allow viewing by multiple workers at a time (Yeh et al., 2012). The main purpose of using these methods is to reduce the dependence on paper. Whilst this would appear to be a perfect solution, there is currently no method for automatically determining the optimum visualisation parameters for the drawing (colour, line thickness etc.), based on the colour and texture of the surface being projected onto. This is crucial because projecting lines of one colour onto a surface of the same colour would render them completely invisible. Rough surface textures could also obscure thin lines and allow detail to be missed, or even make the entire drawing impossible to read at all. To solve this, colours and thicknesses could be adjusted for each surface in the original drawing, but this adds extra tasks for the designer and would be impractical for the worker to do on site. Also colours of surfaces could change between uses, for example applying render or painting a wall. A research has been done into optimum colour schemes for see-through AR headset displays (Gabbard et al., 2005), trying to optimise the viewing of text on the display in general urban outdoor environments. However only text recognition was investigated in the study and projection based displays were not investigated. These displays function quite differently compared to the see-through displays used in the study. To tackle the limitation, this paper presents a system that is able to automatically change the visualisation parameters such as line thickness and line colour based on the colour and texture of the current surface to make drawings visible under any conditions. 


\subsection{Functional Requirements of a New Solution}

The use cases of the final product based on the proposed system are quite varied, from simple projection of points for cabinet installation or other day-to-day job site applications (Figure 1), to projection of entire HVAC systems on top of the already installed system for inspection purposes. It is possible to use the system in a master/slave configuration with the projector communicating with a separate positioning unit that has a known position (useful for large areas), or in standalone mode using positioning targets mounted on the building, allowing the system to be used in more confined areas for applications such as tiling layouts in a small room.

The projector is envisaged to be used over a relatively small range of distances from the surface to maintain the maximum possible brightness of the projected image. In addition, when using the system to transfer markings onto surfaces, the operator will need to keep the same distance from the surface. For these reasons, increasing the size of text and lines when the projector is far from the surface will not be investigated in this study. The system will be used on floors, walls and ceilings, in both external and internal lighting conditions. As such, the visualisation solution needs to function reliably on a large range of different surfaces and under a variety of conditions to be suitable for the system.

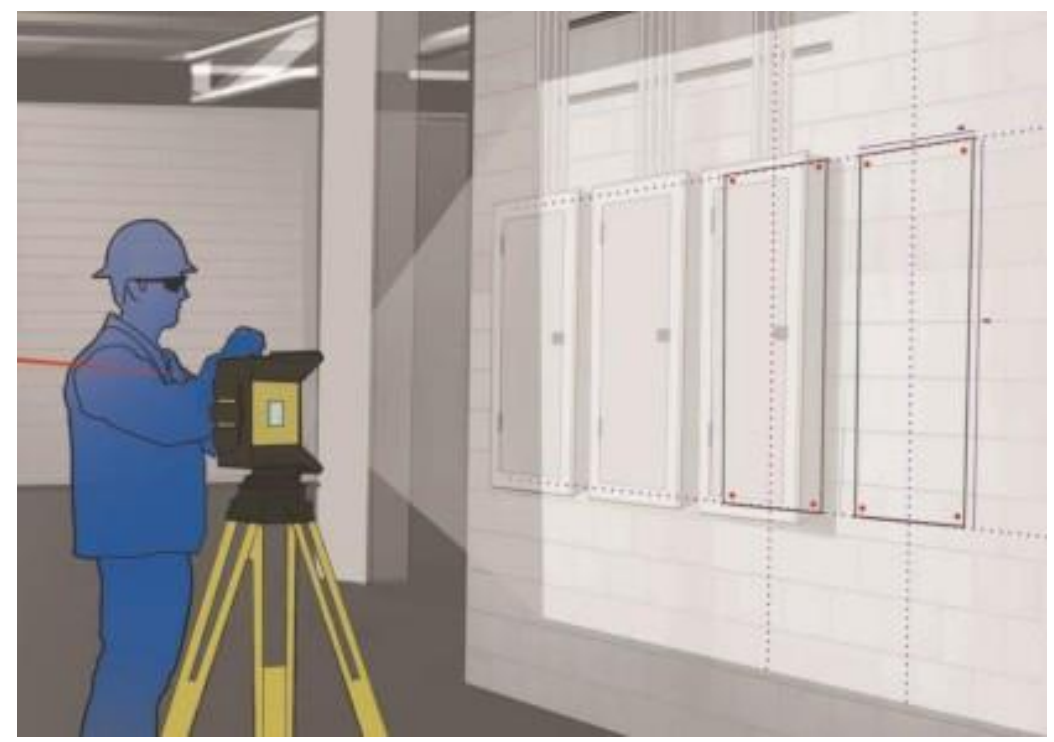

FIG. 1: Usage example of the proposed system.

To work effectively with the proposed software and hardware system, the visualisation solution should:

- Allow drawings to be visible when projected onto any uniform surface, but be especially effective on common construction site surfaces;

- Be responsive and accurate under a variety of lighting conditions;

- Consider both surface colour and texture when optimising the display

- Be stable with regards to the movement of workers in the camera view, and not take items in the camera view that are not part of the surface into account (workers' clothing, tools etc.)

It is noteworthy to mention that non-uniform or round surfaces are out of scope of this paper and could be interesting directions for future research. Our proposed visualisation solution is the focus of investigation for this paper, which will decrease the time spent on reading drawings, marking and measuring, and so intends to improve worker productivity and on a larger scale to benefit the overall productivity of the construction industry.

\section{PROPOSED SOLUTION}

\subsection{Overview}

\subsubsection{Software Overview}

The proposed solution for the software algorithm consists of two parts as shown in Figure 2: (1) the optimisation run that computes and updates the visualisation parameters, and (2) the detection loop which runs continuously 
and checks if the optimisation run needs to be triggered or not. These two parts work together to make the system both responsive and robust to environmental changes and user actions.

\section{Optimization Run}

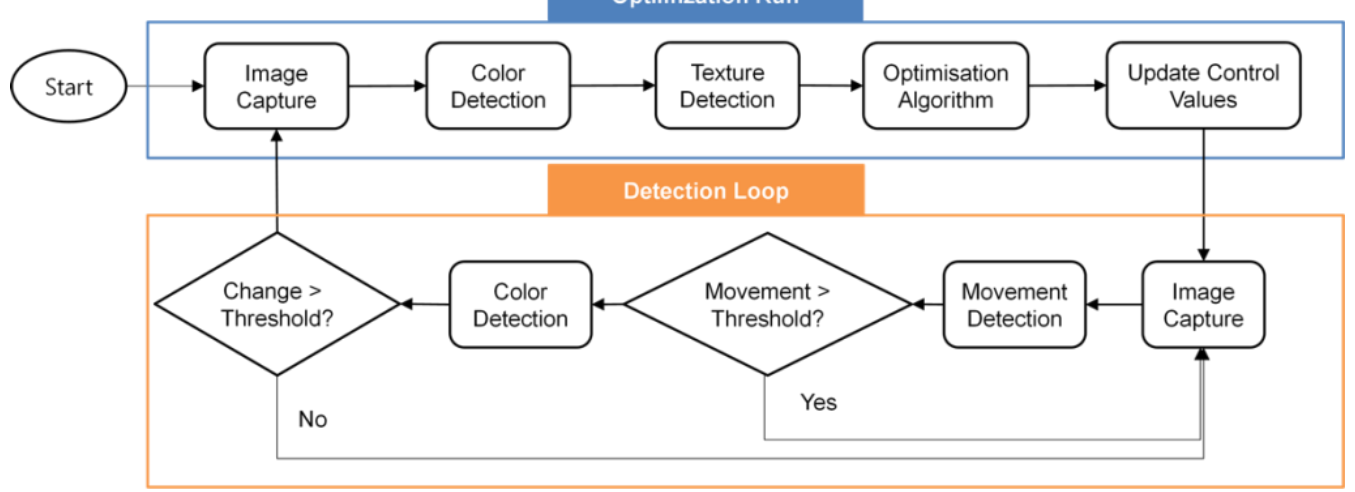

FIG. 2: Overview of the proposed software system.

First, the optimisation run is composed of the following steps: (1) the user takes an image of the surface; (2) the software computes its average colour; (3) it detects the surface texture; and (4) runs the optimisation algorithm based on the computed colour and texture and finally updates the display and the new control values. It is worth mentioning that updating the display is not instant since images should first be captured, then processed and the display parameters should be updated which briefly disrupts the display of the drawing. Therefore, it is important to check whether the environment has changed such that the display needs to be updated. Another important functional requirement of the system is that it should not be changed continuously due to tiny changes in lighting, since this would be disruptive to the user. This technical requirement is achieved by the detection loop.

The detection loop consists of two parts: (1) movement detection and (2) colour detection. First, movement is detected to ensure that the display should not be changed if the worker who marks the surface or takes measurements is in front of the camera. This is because their clothing will be a large part of the captured image, potentially affecting the average background colour. We also assume that the device is being operated by one person. If no movement is detected, the colour of the background is computed and compared to the average colour of the control image which was captured last time. If this average colour differs by a certain amount from the control image, the optimisation run then is triggered and the display is updated.

Further improvements of this solution could be to combine the movement detection output with feedback from the position sensors mounted in the system. However, the system should function as described and detect movement and background changes independent of any movement of the projector or sensors, as large lighting changes could impact the visualisation parameters even if the system and worker position has not changed. Furthermore, movements (translation, rotation) of the system should not necessarily impact the parameters, for example if the background is the same from one position to the next.

\subsubsection{Hardware Overview}

The hardware system consists of two parts: (1) a micro projector and (2) an industrial vision camera which were selected based on their compliance to the user requirements of the system defined above. The projector is an Optoma ML750ST, which is a short throw ultra-compact LED DLP projector. It is highly suited to this application because it has a very short throw lens allowing the system to be used in tight spaces. It is also very compact so that it can be used for integration into a finished product. In terms of power consumption, due to the efficiency of LED light source, the device can be powered from a battery pack, having a high potential for being used on construction sites. Moreover, it is extremely bright so it is not affected by ambient light or external conditions, and the LED technology gives very good colour representation. The camera is an IDS uEye UI-3580-ML (uEye ML Camera, 2021), a 5 mega-pixel USB 3.0 colour camera designed for machine vision. We also used its control software, uEye Cockpit, which (a) allows straightforward adjustment of the camera parameters on the fly, (b) can automatically adjust the camera settings in real time to adapt for ambient lighting conditions and (c) can maintain a consistent image brightness. 
The software solution itself was implemented as a plugin for Trimble SketchUp (Sketchup, 2021), a well-known 3D modelling program as mentioned in the literature review. Trimble SketchUp has a rich API written in Ruby (Ruby, 2021). This allows straightforward control of all display colours and line thicknesses, which would otherwise be very difficult to achieve by manipulating JPEG drawing images. We implemented our solution as a software prototype and communication between the plugin and uEye Cockpit has been achieved through VBScripts for speed and ease of testing. In addition, the numerical operations were calculated in MATLAB.

\subsection{Proposed System Details}

\subsubsection{Input image captures}

We used low image resolution with 288 pixel by 210 pixel for the captured images of our solution. We selected this resolution since detail is not required to compute an average colour and texture. Higher image resolution would been slower processing and lower frame rate which would not allow the camera to respond fast to sudden light changes (e.g. when the background of the display changes). We used uEye Cockpit to crop the image down to a small area of interest limited to the drawing window only, so the colour of the menus and surrounding window is not considered. We also used a feature of uEye Cockpit for automatic gain compensation in order to capture images with consistent brightness. This is noteworthy so that filtering parameters and thresholds in the optimisation loop are not sensitive to background colour changes. As a result, our colour detection algorithm gives consistent results under different ambient conditions. We project the image at maximum brightness irrespective of the conditions to always give the best output and the loss of ambient brightness information is not important. Further enhancements of our proposed solution could recover the ambient brightness from the gain value in the metadata of each image.

\subsubsection{Background Colour Detection}

The captured image is the projected drawing on the surface and is the input of the detection loop, which detects surface changes. We first detect the background colour of an image using a modal average in order to remove any effect of static objects in the camera view (i.e. worker's hands, mortar between bricks). The mode value of the image is insensitive to these artifacts as opposed to the standard mean value of the average pixel colour. Figure 3 illustrates the difference between a mode and a mean average colour detection on a textured concrete background, and Figure 3(b) shows the even larger difference when a worker's hand is in the camera view as well.

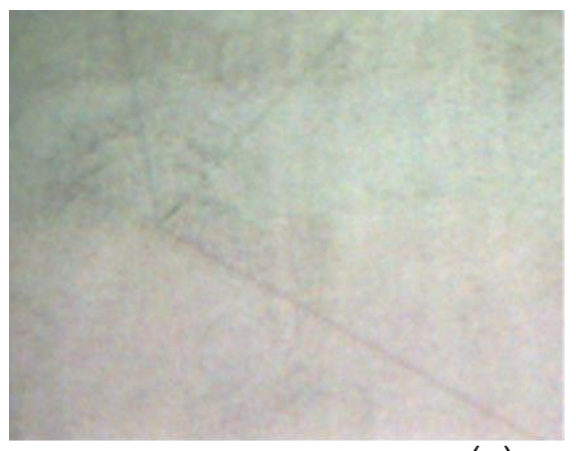

Mean (RGB): 190, 193, 181

(a)

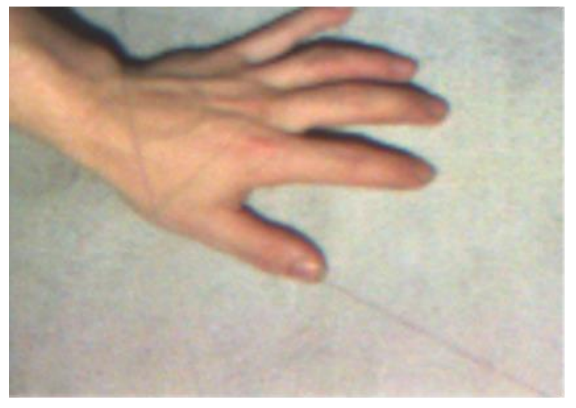

Mean (RGB): 190, 166, 150

(b)

Mode (RGB): 210, 210, 200
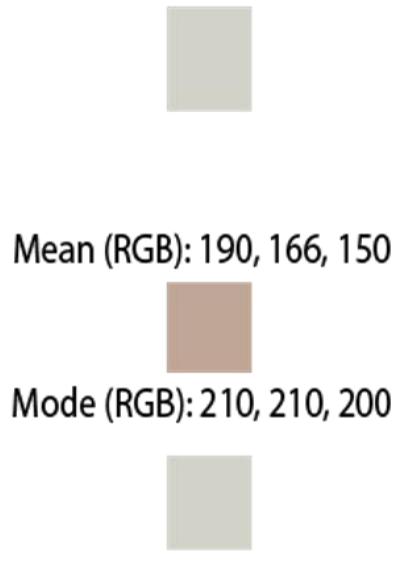

FIG. 3: Mean and modal colour averages of concrete background: (a) without and (b) with worker's hand in camera view. 
A limitation of the modal average is when used on small images. The primary reason for this is that a modal average coupled with the small number of pixels can result in a very small number of pixels having the exact same colour. This gives inconsistent results on some images. For this reason, the pixel colours are first binned by rounding the R, G and B components to the nearest multiple of 10, resulting in only around 16,000 possible colours. This binning technique gives much more reliable results, especially on textured backgrounds with a relatively large range of pixel colours.

\subsubsection{Background Texture Detection}

Obtaining a measure of surface roughness is crucial for successfully optimising the display as thin lines on rough surfaces such as brick and concrete can be easily obscured. However, it is important that lines remain as thin as possible so that the accuracy of the projected drawings on the surface is maintained. We calculate the image surface roughness based on the background image and then feed this as input to the optimisation algorithm where it is used as a scaling factor for the line thickness.

We compute texture by calculating the variance of pixel intensity over a certain distance from the centered pixel. We designed an algorithm to give large output on surfaces such as concrete and brick whilst ignoring lighting and other artefacts on smooth, single colour surfaces. Since only pixel intensity is used for this calculation, images are first converted to 8 bit gray scale by discarding the colour information. To reject noise and large scale lighting changes across the image, the brightness and contrast are increased by 50 and 150 respectively to highlight any small-scale texture information while discarding gradual changes due to lighting effects. Although we chose those values based on our experiments on test images of various backgrounds, note that the values can vary with different lighting and image conditions. We increase brightness by simply adding a constant to the $\mathrm{R}, \mathrm{G}$ and $\mathrm{B}$ values of each image pixel and then normalizing them. Similarly, we increase brightness by first calculating the correction factor $\mathrm{F}$ from the desired increase in contrast $\mathrm{C}$ and then use it on the red, green and blue components separately to move the overall pixel colour away from gray (128) and then normalize the values.

We follow the approach proposed by Cao et al., 2010 and the equations below:

$$
F=259 *(C+255) /(255 *(259-C))
$$

As an example, the adjusted colour is computed using the formula:

$$
r^{\prime}=259 *(r-128+128)
$$

Figure 4 shows the result of the algorithm that highlights the image details (Figure 4(a)) by increasing the brightness and contrast of the textured background image compared to the result on the non-textured background image (Figure 4(b)) showing a completely white output.

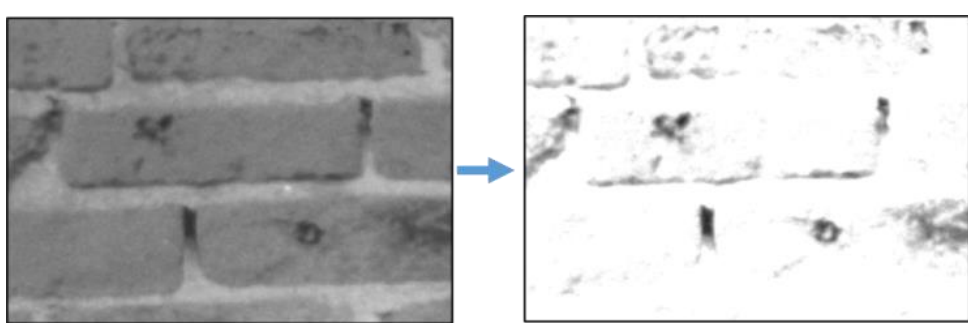

(a)

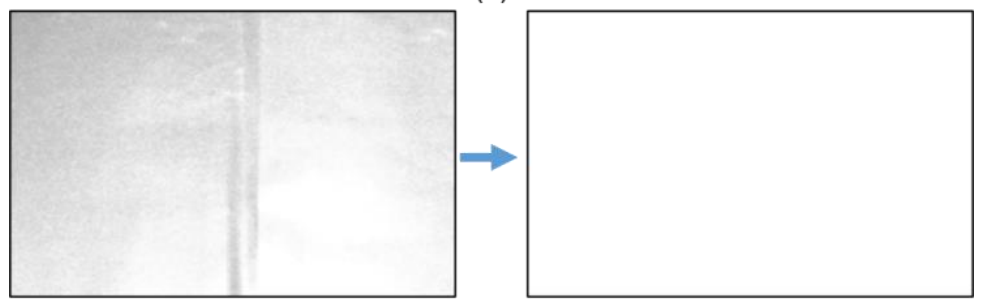

(b)

FIG. 4: Background text detection on: (a) textured background after brightness and contrast adjustment; and (b) non-textured background after brightness and contrast adjustment. 


\subsubsection{Optimisation algorithm}

The optimisation algorithm aims to find the display parameters that maximize the view clearness of projected drawings to the users. To this end, it takes the computed values for the background colour and texture, and uses them to adjust the available display parameters of the drawing for optimum viewing on the current surface. Our optimized parameters are: (a) the background colour, (b) face colour, (c) line colour and (d) line thickness of the drawing. The algorithm is based upon the theory that the colour having the most contrast to another has a hue that is 180 degrees different to the first colour.

We first optimize the average colour of the background using a colour correction algorithm that accounts for camera characteristics. We found the coefficients of this correction after experimentation as we will discuss in the Results Section. We then set the background colour as the corrected colour. We then optimize the face colour by adapting it to the lightness of the background colour in an attempt to provide more contrast between the background and line colours without adding yet another colour into the display. We set the face colour to white for surfaces with a lightness of less than 0.65 (dark surfaces) whereas we set it to black for surfaces with lightness above 0.65 (light surfaces) after experimentation. Note that although we chose the threshold based on our experiments on test images of various backgrounds, the threshold can vary with different lighting and image conditions. For the line colour, we use an HSV (Hue, Saturation, Value) compliment technique to achieve maximum contrast against the background since the HSV colour space separates chromatic and achromatic components (Kavitha et al., 2011). We first saturate the background colour and then add 180 degrees to the hue value, which gives a fully saturated opposite colour. This is then set as the line colour for the drawing. We finally calculate the line thickness using the standard deviation from the texture detection function.

\subsubsection{Movement detection}

The movement detection system is crucial to the reliability of the system as the display should not be updated whilst the hardware is being moved across the site but updated only when the device is stationary in its new position. Workers moving in front of the camera view are also likely to change the average colour seen by the camera, so it is essential to detect their movement without triggering the optimisation run that would cause visualisation colour errors. It is also important that the detection algorithm runs as quickly as possible as it is the largest, most complex part of the detection loop and is always running unlike the colour detection algorithm. For these reasons, the algorithm has been simplified as much as possible to limit the features to only those necessary for the operation of the system.

The first part of the movement algorithm compares the last captured image with the control image, in order to perform background subtraction. Each pixel is compared in turn, and if the R, G and B components of the pixel in the current image are within a certain threshold of the control image, the pixel is considered background and turned black. If they exceed the threshold limits, the pixel is considered foreground and turned white. It is important to note that a control image is the image capture taken with the drawing projected, immediately after the background/line colours are changed.

Figure 5 shows the result of this background subtraction on a background with a worker's hand in the camera view, as if the worker was marking a point on the surface. The resulting black and white image reduces the amount of information needed to be processed by the following stages.

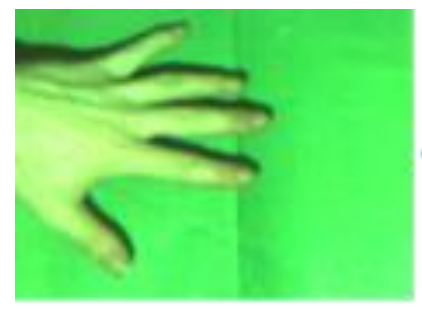

(a)

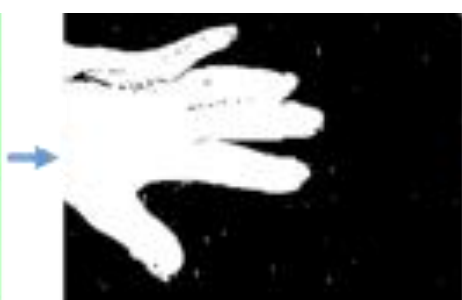

(b)

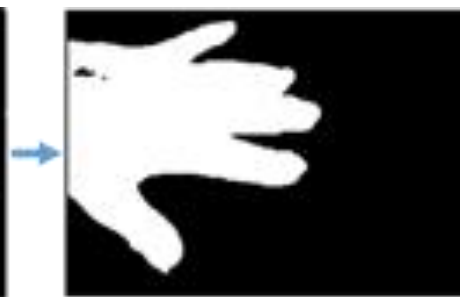

(c)

FIG. 5: (a) Original image capture, (b) background subtraction with worker's hand in camera view (before filtering) and (c) worker hand after filtering. 
Camera sensor noise and lighting effects create artifacts between foreground and background pixels. Therefore, image filtering (blob filter) is applied to remove camera sensor noise and lighting effects based on the following assumptions: (1) foreground features of interest are assumed to be large blocks of pixels (e.g. hand, body); (2) singleton white or black pixels are anomalies or noise. Figure 5(b) shows the background removal with no filtering and Figure 5(c) shows the filtered image. It is important to note that blob filtering is fairly computationally intensive $\left(\mathrm{O}\left(\mathrm{n}^{2}\right)\right)$ and gray scale images have been used.

If a number of matching pixels in the blob is below the threshold, the pixel is classified as an anomaly and is flipped after all the other pixels have been tested. The image is filtered in two passes - for the first pass, white anomaly pixels are filtered out and for the second one, black anomaly pixels give a very clean output image.

The resulting filtered foreground image is then compared against the previous filtered foreground image from the last run of the detection loop by counting the number of different pixels between the images. Figure 6 shows the result of when the worker's hand is moved across the camera view. It is noteworthy that the white pixels denote differences between the first and subsequent image captures. The number of differing pixels is then compared to a threshold (2,000 pixels) and if this is exceeded, movement is detected.

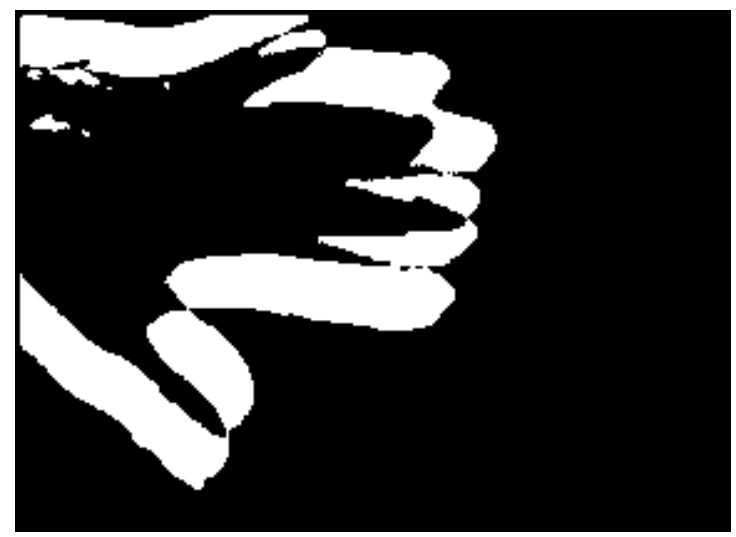

FIG. 6: "Moving" pixels between two frames of a worker moving their hand across the camera view.

\section{VALIDATION AND RESULTS}

\subsection{Description}

We designed and conducted a user test to objectively measure the effectiveness of the system on a variety of surfaces. Since this system is designed to be used on surfaces commonly found at construction sites, this study conducted objective testing using materials identified to be common for the use cases of the system, namely: (1) brick, (2) concrete block, (3) concrete, and (4) Oriented strand board (OSB). For ease of testing, the backgrounds were printed out on A3 paper with the textures scaled to match real world material samples, e.g. brick size.

In order to assess the effectiveness of the system, four layouts were tested:

- White background and faces, black lines (control colour scheme without optimisation);

- White background and faces, blue lines;

- The system with colour optimisation only;

- The system with colour and line optimisation.

For each test, the test subject was asked to find and mark the locations of 10 small holes as shown in Figure 7 projected onto the surface in a short time frame. The locations of the holes were randomised for each background and optimisation scheme combination. The holes were sized such that it was difficult (but not impossible) to see them with the control colour schemes on the non-control test surfaces. The time taken to complete this for each surface and optimisation technique was recorded. Each test ran for a maximum of 60 seconds, as the test subjects were not always able to find all the holes with every colour scheme and background combination. In this study, we used eight different test subjects. The white background and white/black colour scheme served as a baseline reading, with the hypothesis that if the time taken to find the holes on the coloured surfaces with the optimisation 
system in place was the same as that for the control case, the system was performing sufficiently well and could not be optimised any further. The eight test users' reading times for each test combination were averaged to give a mean time for each combination.

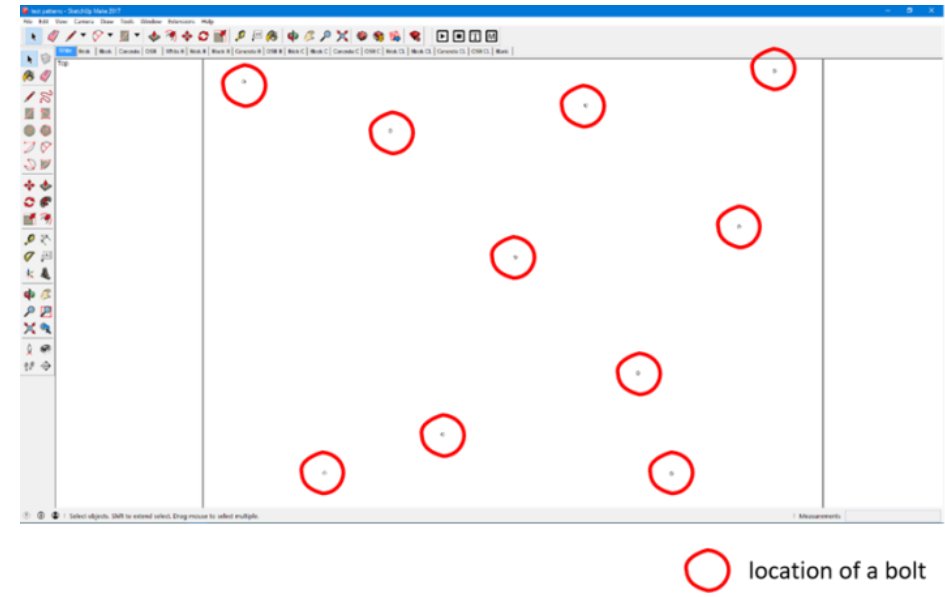

FIG. 7: Example bolt hole test pattern (red circle are only used in this graph to illustrate the position of the holes and they were not part of the pattern).

\subsection{Results and findings}

Figure 8 shows the visualisation results of small bolt holes, without any optimisation, with just colour optimisation and finally with both colour and line optimisation, which increases the visibility of the projected drawings. Table 1 and 2 show the time taken for the 8 subjects to find all the 10 bolt holes and we present the average results for all users in Figure 9. The error bars shown on Figure 9 denote the minimum and maximum times taken by all users to find the holes for a given test combination.
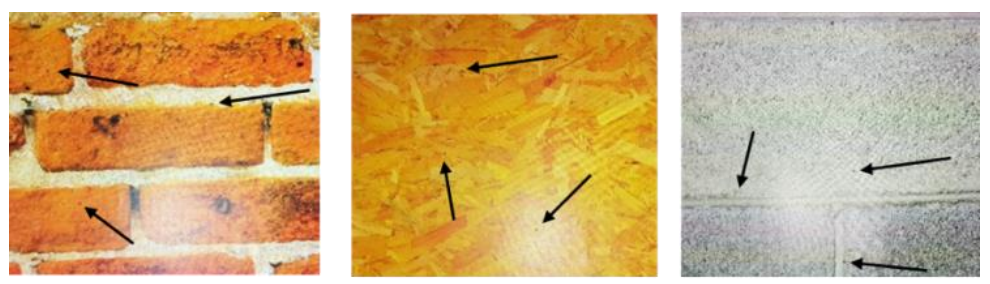

Without any optimization
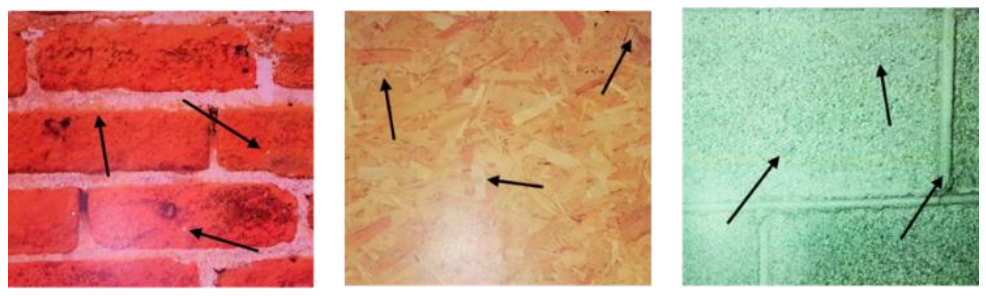

With color optimization only
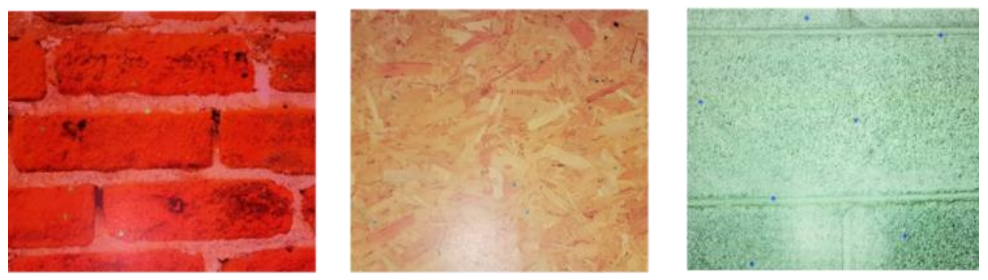

With color and line thickness optimization

FIG. 8: Visualisation results of bolt holes projection: (a) without any optimisation; $(b)$ with colour optimisation only; and (c) with colour and line thickness optimisation. 
TABLE 1: Time taken to find the 10 bolt holes (in seconds).

\begin{tabular}{cccccc}
\hline \multicolumn{5}{c}{ Control (B+W) } \\
$\begin{array}{c}\text { Text } \\
\text { Subject }\end{array}$ & White & Brick & Block & Concrete & OSB \\
\hline 1 & 10 & 60 & 60 & 60 & 47 \\
2 & 10 & 60 & 60 & 60 & 39 \\
3 & 10 & 60 & 60 & 60 & 32 \\
4 & 9 & 60 & 60 & 60 & 60 \\
5 & 10 & 60 & 60 & 60 & 41 \\
6 & 10 & 60 & 60 & 60 & 40 \\
7 & 8 & 60 & 60 & 60 & 45 \\
8 & 9 & 60 & 60 & 60 & 42 \\
\hline & & Colour Compensation & & & \\
\hline Text & White & Brick & Block & Concrete & OSB \\
Subject & & & & & \\
\hline 1 & 10 & 28 & 22 & 30 & 30 \\
2 & 10 & 22 & 20 & 25 & 18 \\
3 & 10 & 39 & 13 & 14 & 27 \\
4 & 9 & 21 & 15 & 14 & 23 \\
5 & 10 & 25 & 16 & 18 & 24 \\
6 & 10 & 22 & 15 & 27 & 25 \\
7 & 8 & 24 & 19 & 21 & 30 \\
8 & 9 & 26 & 14 & 17 & 29 \\
\hline
\end{tabular}

TABLE 2: Time taken to find the 10 bolt holes (in seconds).

\begin{tabular}{cccccc}
\hline \multicolumn{5}{c}{ White/Blue } \\
$\begin{array}{c}\text { Text } \\
\text { Subject }\end{array}$ & White & Brick & Block & Concrete & OSB \\
\hline 1 & 9 & 47 & 25 & 45 & 40 \\
2 & 10 & 60 & 28 & 15 & 55 \\
3 & 8 & 50 & 16.7 & 13 & 32 \\
4 & 7 & 60 & 16 & 19 & 22 \\
5 & 8 & 49 & 19 & 33 & 34 \\
6 & 10 & 44 & 23 & 16 & 30 \\
7 & 7 & 51 & 27 & 39 & 39 \\
8 & 8 & 47 & 22 & 30 & 40 \\
\hline & & Colour + Line Compensation & & & \\
\hline Text & White & Brick & Block & Concrete & OSB \\
Subject & & 19 & 18 & 14 & 16.5 \\
\hline 1 & 10 & 14 & 12 & 12 & 11 \\
2 & 10 & 11 & 9 & 10 & 7 \\
3 & 10 & 12 & 8 & 7 & 7 \\
4 & 9 & 16 & 14 & 11 & 8 \\
5 & 10 & 14 & 17 & 13 & 10 \\
6 & 10 & 15 & 17 & 12 & 12 \\
7 & 8 & 15 & 15 & 10 & 9 \\
\hline 8 & 9 & & & & \\
\hline
\end{tabular}




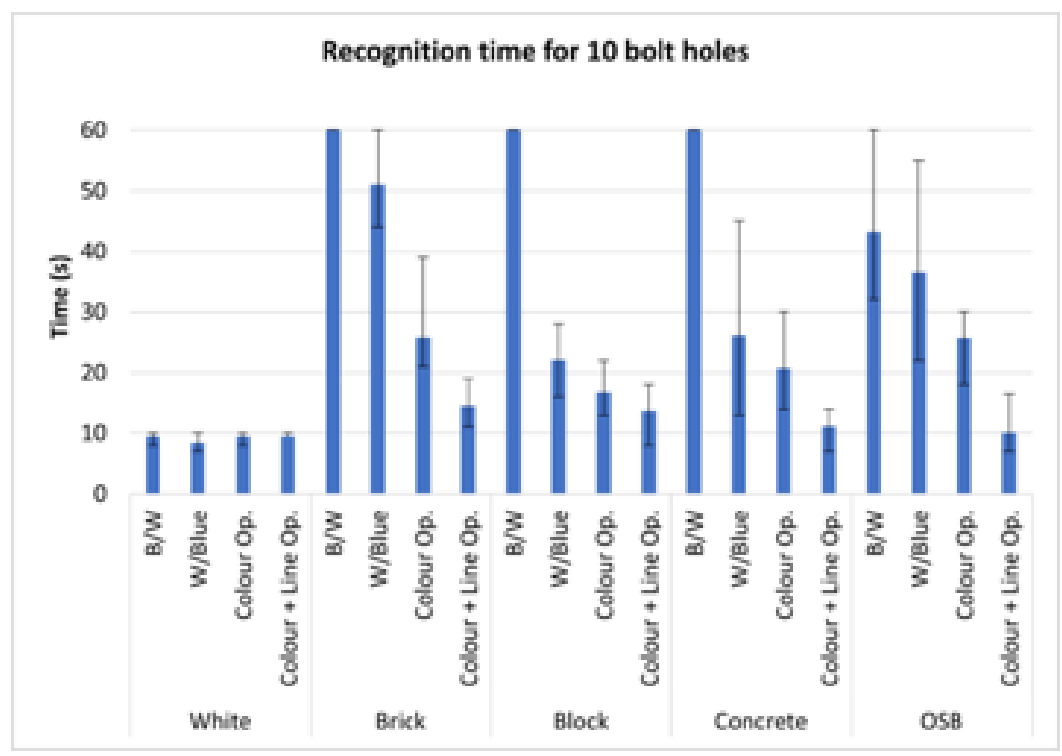

FIG. 9: Final results from user tests with its maximum and minimum time for each case (a total of 20 cases)

First, the results show the level of importance of solving this optimisation problem. Regarding the control black/white scheme, every user could quickly identify the holes on a white background. However, on the realworld surfaces $75 \%$ the subjects were not able to find all the holes. This would be highly detrimental to a final project as not only would it take more time and possibly decrease the worker's efficiency compared to standard methods, but missing parts of a drawing can result in mistakes leading to costly rework operations.

It is very clear from the data that overall the system had an obvious effect in decreasing the time taken to find the 10 holes on all the test surfaces, which succeeds in making drawings easier to read on both coloured and textured surfaces. The colour optimisation alone reduced the time taken to read a drawing to around half of the time needed for the black and white control colour scheme. If this is coupled with the line optimisation as well, this time was reduced even further and this result was consistent across all tests.

For the control tests on the white background, the users' times were very consistent at around 10 seconds for finding all the holes, with a very low variance between the maximum and minimum times. During the tests, 10 seconds was the time taken to physically circle the holes on the surface and no time was taken visually searching for the holes. Based on this observation, we can assume that the system needs at least 10 seconds for the workers to start working on tasks using this system.

The white and blue colour scheme provided a noticeable improvement on all the test surfaces, which was the same result obtained by Gabbard et al. which compared colour schemes for see-through AR displays (Gabbard et al., 2005). This is likely due to "black" colour on the projection making it difficult to identify occlusions in that background (as in see-through AR displays). Therefore, projecting blue instead of no colour gives a better contrast for the user. This suggests the white/blue colour scheme as a satisfactory and straightforward first step to apply instead of white/black for any surface and drawing.

The colour optimisation tests with standard line thicknesses improve the results even further on all surfaces, making especially good improvements on the brick and OSB backgrounds. The block and concrete backgrounds were improved slightly, however not at a significant amount. This is likely since the results of the algorithm on these surfaces are in fact very similar to the white/blue colour scheme as the surfaces are a light grey. This therefore gave a much less noticeable effect compared to the brick and OSB, which were a much more saturated orange colour.

The final test using the full algorithm gave the best results, providing especially good improvements on the highlytextured brick and OSB surfaces. The average times for the surfaces under this scheme were the same (OSB, concrete) or very close (block, brick) to the control time of 10 seconds on the white background, showing the system performed very effectively and has negated the effect of the background surfaces on the visibility of the projection. 
In terms of statistical significance, the minimum/maximum times taken for each test shown by the error bars (Figure 9) are quite large in some cases (i.e. concrete surface, white/blue colour) - this was normally due to a single user spending a very large amount of time finding the last hole, or not being able to find a hole at all. Theoretically a test should be designed such that small outliers such as this should not affect the results. However, the comparatively large error bars actually give more insight in this case. For all the surfaces apart from the concrete block, the error bars decrease in size significantly as the average time decreases. This suggests that the optimisation algorithms not only reduce the average time spent, but they also increase the consistency of the results between users, with the lower average times having a much smaller variance.

A t-test was conducted on the results to assess the confidence level between the control tests (black and white) and other optimisation strategies on each background. Table 3 shows the t-test results. All the tests had a confidence level of over $99.9 \%$, with the exception of the white/blue scheme on the OSB background which improved the times with a confidence level of $80 \%$. A t-test was also performed between the colour optimisation only, and the colour and line optimisation on each background to get a confidence level of the improvement after adding the line thickness enhancement part of the algorithm. Experiments with all backgrounds had a confidence level of 99.9\% apart from the concrete block background which had a confidence level of $95 \%$.

TABLE 3: $t$-test results of user tests

\begin{tabular}{lllcl}
\hline \multirow{2}{*}{ Surface } & Control Group & Test Group & C-value & Limit \\
\hline \multirow{2}{*}{ Brick } & Black/white & White/Blue & -4.28 & $99.9 \%$ \\
& Black/white & Colour & -16.66 & $99.9 \%$ \\
& Black/white & Colour + Line & -52.54 & $99.9 \%$ \\
& Colour & Colour + Line & -13.13 & $99.9 \%$ \\
\hline Block & Black/white & White/Blue & -23.64 & $99.9 \%$ \\
& Black/white & Colour & -38.28 & $99.9 \%$ \\
& Black/white & Colour + Line & -34.70 & $99.9 \%$ \\
& Colour & Colour + Line & -2.25 & $95.0 \%$ \\
\hline \multirow{2}{*}{ Concrete } & Black $/$ white & White/Blue & -7.86 & $99.9 \%$ \\
& Black $/$ white & Colour & -18.38 & $99.9 \%$ \\
& Black $/$ white & Colour + Line & -63.79 & $99.9 \%$ \\
& Colour & Colour + Line & -12.56 & $99.9 \%$ \\
\hline OSB & Black/white & White/Blue & -1.52 & $80.0 \%$ \\
& Black/white & Colour & -5.44 & $99.9 \%$ \\
& Black/white & Colour + Line & -10.79 & $99.9 \%$ \\
& Colour & Colour + Line & -5.10 & $99.9 \%$ \\
\hline
\end{tabular}

\subsection{Comparison with initial requirements}

Whilst the system clearly improves the visibility of the drawings on the test surfaces, the system also has requirements to fulfil in order to add value to the construction industry as discussed in the introduction:

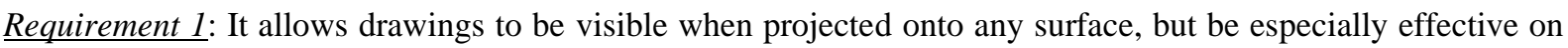
common construction site surfaces

This requirement has clearly been met as recognition times for the most common construction site materials were dramatically improved by using the system compared to control cases, and initial testing was done on the solid colour printouts allowing the system to function on any background to improve the visualisation to some degree.

\section{Requirement 2: It is responsive and accurate under a variety of lighting conditions}

The system is insensitive to external lighting conditions by using the high brightness of the projector to illuminate the surface when capturing images, so it is not dependent on ambient conditions. 


\section{Requirement 3: It considers both surface colour and texture when optimising the display}

Colour and texture are both considered, and combined to create the final display parameters for the given surface. The test results show that the line thickness adjustment further decreases the comprehension time when compared to using the colour optimisation alone.

Requirement 4: It is stable with regards to the movements of workers in the camera view, and not consider items that are not part of the surface as foreground in the camera view

The movement detection algorithm effectively determines when items are not part of the surface in the camera view, and the optimisation does not take them into account. The colour detection also uses a modal average to remove the effects of small foreign objects in front of the camera and provides a more reliable result for surfaces that might have multiple major colours such as bricks and mortar between them. Overall, the proposed system fulfills all these requirements and would be valuable for the construction workers to use.

\section{CONCLUSIONS AND FUTURE WORK}

Current practice in implementing the task of reading drawings on job sites has efficiency and cost problems, some of which include keeping track of versions and distributing change orders. Although recent products utilising laser projectors have attempted to address the issue of drawing comprehension, they only allow projection in red or green at a single brightness level, which could cause visualisation problems depending on the surface to be projected on and the ambient conditions. To tackle this problem, we developed a prototype system in this study to automatically change the visualisation parameters such as line thickness and line colour based on the colour and texture of the current surface so that drawings are visible under any conditions. The proposed system consists of software and hardware, and the software algorithm consists of two modules: (1) the optimisation run that computes and updates the visualisation parameters and (2) the detection loop which runs continuously and checks whether the optimisation run needs to be triggered or not. In order to investigate the feasibility of the proposed system, the system was tested on 8 subjects with 4 background surfaces commonly found on site, with the test subjects timed to find 10 bolt holes projected onto the surface using the optimisation system, which was then compared to a control case of black lines projected onto a white background. The final test using the full algorithm gave the best results, providing especially significant improvements on the highly-textured brick and OSB surfaces. The average times for the surfaces under this scheme were the same or very close to the control time of 10 seconds on the white background, demonstrating that the system performed very effectively and has negated the effect of the background surfaces on the visibility of the projection. In summary, the system allowed users to complete the task on the real-world backgrounds in the same time as the control case, with the system resulting in up to a $600 \%$ decrease in recognition time on some backgrounds. From these results, it is envisaged that the proposed visualisation solution can improve the productivity of the worker. The academic contribution of this study is the development of a solution that adjusts the visualisation parameters of the displayed information based on the surface being projected onto to make drawings visible.

However, there are some limitations in the current study that will be the directions for future study. First, as the projected images could be affected by the texture and colour of the surface to be projected, further study on geometric calibration to project drawings on the right region at the construction site is necessary. Second, userdefined thresholds were used for the optimization and detection algorithms, which might be not robust in order to apply the proposed method on real sites. Thus, further analysis on how to determine the threshold is needed. In addition, future research directions could be to use deep learning methods to automatically segment the foreground from background. Our solution and extensions of it have the potential to benefit the overall productivity of the construction industry by decreasing the time spent on reading drawings, marking and measuring.

\section{REFERENCES}

Alfano P.L., Michel G.F., Restricting the field of view: perceptual and performance effects, Perceptual and Motor Skills, 1990, 70(1), 35-45.

Arthur K.W. Effects of Field of View on Performance with Head-Mounted Displays. Ph.D. Dissertation, Department of Computer Science, UNC Chapel Hill, April 2000.

Autodesk 360. Available online: https://a360.autodesk.com/ (accessed on 24 May 2021). 
Bae H., Golparvar-Fard M., White J. High-precision vision-based mobile augmented reality system for contextaware ar- chitectural, engineering, construction and facility management (AEC/FM) applications. Visualisation in Engineering 2013, 1, https://doi.org/10.1186/2213-7459-1-3.

Bandyopadhyay D., Ramesh R., Henry F. Dynamic shader lamps: Painting on movable objects in: Proceedings of the IEEE and ACM International Symposium on Augmented Reality 2001, IEEE Computer Society, USA.

Cao G., Zhao Y., Ni R., Forensic estimation of gamma correction in digital images in: 2010 IEEE International Conference on Image Processing, IEEE, 2097-2100.

Chi H., Kang S., Wang X., Research trends and opportunities of augmented reality applications in architecture, engineering, and construction. Automation in Construstion 2013 33, 116-122, https://doi.org/10.1016/j.autcon.2012.12.017.

Cuperschmid A.R.M., Grachet M.G., Fabrício M.M., Development of an augmented reality environment for the assembly of a precast wood-frame wall using the BIM model, Ambiente Construído 2016 16, 63-78, https://doi.org/10.1590/s1678- 86212016000400105.

Dalsgaard P., Halskov K., 3D projection on physical objects: design insights from five real life cases in: Proceedings of the SIGCHI conference on human factors in computing systems, 2011, ACM.

Feiner S., Macintyre B., Seligmann D. Knowledge-based Augmented Reality. Communications of the ACM 1993, 36(7), 53-62.

Funk M., Bachler A., Bachler L., Kosch T., Heidenreich T., Schmidt A., Working with augmented reality: A long-term analysis of in-situ instructions at the assembly workplace, in: 10th International Conference on Pervasive Technologies Related to Assistive Environments, 2017, 222-229, https://doi.org/10.1145/ 3056540.3056548.

Gabbard J.L., Swan J.E., Hix D., Schulman R.S., Lucas J., Gupta D. An Empirical User-based Study of Text Drawing Styles and Outdoor Background Textures for Augmented Reality. Proc. IEEE Virtual Reality 2005, https://doi.org/10.1109/VR.2005.1492748.

Golparvar-Fard M., Pena-Mora F., Savarese S., D4AR-a 4-dimensional augmented reality model for automating construction progress monitoring data collection, processing and communication, Journal of Information Technology in Construction (ITCon) 2004, 14, 129-153.

Hou L., Wang X., Truijens M., Using augmented reality to facilitate piping assembly: an experiment-based evaluation, Journal of Computing in Civil Engineering 2013 29(1), https://doi.org/10.1061/(ASCE)CP.1943-5487.0000344.

Hubert D., Living in a world transformed: perceptual and performatory adaptation to visual distortion, Academic Press, 1982.

Kavitha C., Rao B.P., Govardhan, A., An efficient content based image retrieval using color and texture of image sub blocks, International Journal of Engineering Science and Technology (IJEST) 2011, 3(2), 1060-1068.

Kim H., Kang L., AR-based 4D CAD system using marker and markerless recognition method, Procedia Engineering, 2017, 196, 29-35, https://doi.org/10.1016/j.proeng.2017.07.169.

Laser Manufacturer, Z-laser. Available online: http://www.z-laser.com/en/ (accessed on 24 May 2021)

Li X., Yi W., Chi H., Wang X., Chan A.P., A critical review of virtual and augmented reality (VR/AR) applications in construction safety, Automation in Construction, 2018, 86, 150-162, https://doi.org/10.1016/j.autcon.2017.11.003.

Low K.L., Welch G., Lastra A., Fuchs H., Life-sized projector-based dioramas in: Proceedings of the ACM symposium on Virtual reality software and technology 2001, 93-101.

Mine, M.R., Van Baar J., Grundhofer A., Rose D., Yang B. Projection-based augmented reality in disney theme parks, Computer, 2012 45(7), 32-40.

Moore N., Heesom D., Integrating interactive 4D CAD with wide-area augmented reality to enhance on-site construction planning, in: International Conference on Construction Applications of Virtual Reality, 2011, 484-495. 
Office for National Statistics, Labour productivity by industry division. Available online: Link (accessed on 24 May 2021).

Oglesby C.H., Parker H.W., Howell G.A., Productivity Improvement in Construction; McGraw-Hill; 1989; pp.179.

Optoma-ML750ST projector. Specifications available online: https://www.projectorcentral.com/OptomaML750ST.htm (accessed on 2 June 2021)

Park Y.J., Yang Y., Chae S., Kim I., Han T., DesignAR: Portable projection-based AR system specialized in interior de-sign in: IEEE International Conference on Systems, Man and Cybernetics (SMC), Banff, AB, Canada 2017, 2879-2884, doi:10.1109/SMC.2017.8123064.

Rankohi S., Waugh L., Review and analysis of augmented reality literature for construction industry, Visual Engineering 2013, 1(9) (2013), https://doi.org/10.1186/2213- 7459-1-9.

Raskar R., Greg W., Henry F. Spatially augmented reality in: First IEEE Workshop on Augmented Reality (IWAR'98) 1998.

Ruby API. Available online: https://rubyapi.org/ (accessed on 2 June 2021)

Siegl C., Colaianni M., Thies L., Thies J., Zollhöfer M., Izadi S., Bauer F. Real-time pixel luminance optimization for dynamic multi-projection mapping, ACM Transactions on Graphics (TOG) 2015 34(6).

Singh A.R., Delhi V.S.K., User behaviour in AR-BIM-based site layout planning, Int. J. Product Lifecyc. Manag. 2018, 11(3), 221-244, https://doi.org/10.1504/IJPLM.2018.094715.

Takahashi T., Terano T. Big Fat Wand: A Laser Projection System for Information Sharing in a Workspace. Human Interface and the Management of Information 2011, 6772, 403-410.

Trimble SX10 Total Station. Available online: http://www.trimble.com/Survey/Total-Station-SX10.aspx (accessed on 24 May 2021)

Trimble Sketchup. Available online: https://www.sketchup.com/ (accessed on 2 June 2021)

Trimble Yuma 2 Rugged Tablet PC. Available online: Link (accessed on 24 May 2021)

uEye ML Camera. Specifications available online:

https://www.1stvision.com/cameras/sensor_specs/mt9p006_flyer.pdf (accessed on 2 June 2021)

Wang X., Kim M.J., Love P.E., Kang S., Augmented reality in built environment: classification and implications for future research, Automation in Construction 2013, 32, 1-13, https://doi.org/10.1016/j.autcon.2012.11.021.

Wen J., Gheisari M., Jain S., Zhang Y., Minchin R. E. Using Cloud-Based Augmented Reality to 3D-Enable the 2D Drawings of AISC Steel Sculpture: A Plan-Reading Educational Experiment. Journal of Civil Engineering Education 2021 147(3).

Wen M., Kang S., Augmented reality and unmanned aerial vehicle assist in construction management, in: Computing in Civil and Building Engineering, 2014, 1570-1577, https://doi.org/10.1061/9780784413616.195.

Yates J.K., T. Productivity Improvement for Construction and Engineering; ASCE Press; 2014; pp.127.

Yeh K.C., Tsai M.H., Kang S.C. On-Site Building Information Retrieval by Using Projection-Based Augmented Reality. Journal of Computing in Civil Engineering 2012, 26(3), https://doi.org/10.1061/(ASCE)CP.19435487.0000156.

Zaher M., Greenwood D., Marzouk M., Mobile augmented reality applications for construction projects, Constr. Innov. 2018, 18(2), 152-166, https://doi.org/10.1108/CI-02-2017-0013.

Zollmann S., Hoppe C., Kluckner S., Poglitsch C., Bischof H., Reitmayr G., Augmented reality for construction site monitoring and documentation, in: Proceedings of IEEE, 102(2), 2014, 137-154, https://doi.org/10.1109/JPROC.2013.2294314. 